\title{
Estimation of Severe Middle East Respiratory Syndrome Cases in the Middle East, 2012-2016
}

\author{
Justin J. O'Hagan, Cristina Carias, \\ Jessica M. Rudd, Huong T. Pham, Yonat Haber, \\ Nicki Pesik, Martin S. Cetron, Manoj Gambhir, \\ Susan I. Gerber, David L. Swerdlow
}

Middle East respiratory syndrome has been reported among travelers returning from the Arabian Peninsula, where most cases have been recorded. Using data from travelers, we estimated 3,250 $(1,300-6,600)$ severe cases occurred in the Middle East during September 2012-January 2016. This estimate is 2.3-fold higher than the total laboratory-confirmed cases recorded in these countries.

Middle East respiratory syndrome (MERS), caused by MERS coronavirus (MERS-CoV), was first recognized in September 2012 (1). From that time until January 2016, $>1,600$ cases were laboratory-confirmed, and $\approx 600$ deaths have been attributed to the virus (2). Cases have been detected among persons who traveled from the Middle East to 16 countries, and a MERS-CoV outbreak in South Korea introduced by a traveler caused $>100$ cases (3).

Estimates of the epidemic size in the Middle East are required to understand the level of MERS-CoV circulation and the likelihood of MERS-CoV exportations. However, these estimates have not been calculated for $>2$ years, during which time the number of recorded cases has increased by $>15$ times $(2,4)$. We used data from travelers to this region to update estimates of severe MERS cases in the Middle East.

\section{The Study}

We estimated the cumulative number of severe MERS cases in source countries from which laboratory-confirmed MERS-CoV infections were reported by the World Health Organization for nonresident travelers during September 2012-January 2016. The source countries were Saudi Arabia, United Arab Emirates, Jordan, and Qatar (1); only the emirates of Abu Dhabi and Dubai were included in the United Arab Emirates calculations due to a lack of traveler data on the other 5 emirates, but no MERS cases have been reported from these other emirates.

Author affiliations: IHRC Inc., Atlanta, Georgia, USA (J.J. O'Hagan.

C. Carias); Centers for Disease Control and Prevention, Atlanta

(J.J. O'Hagan, C. Carias, J.M. Rudd, H.T. Pham, Y. Haber,

N. Pesik, M.S. Cetron, M. Gambhir, S.I. Gerber, D.L. Swerdlow);

Imperial College London, London, UK (M. Gambhir); Monash

University, Melbourne, Victoria, Australia (M. Gambhir)

DOI: http://dx.doi.org/10.3201/eid2210.151121
All MERS traveler case-patients whose data were used in the analysis were hospitalized because of respiratory symptoms and therefore likely experienced severe disease. However, $30 \%$ of reported MERS case-patients were listed as mildly symptomatic or asymptomatic and were likely missed by the passive surveillance systems that detected severe travel-associated cases (1). Consequently, we used the term "severe" to indicate that our estimates of case numbers are for those with more serious disease.

To estimate the cumulative number of severe cases, we used data for 1) the number of travelers to the 4 source countries, 2) average trip lengths, 3) number of confirmed MERS-CoV infections among travelers to the source countries, and 4) population sizes of source countries. Our estimates are for the period September 2012, when MERSCoV was first identified, through January 2016.

We estimated the number of severe cases in each source country using methods used previously by Cauchemez et al., which assume that travelers and local residents have similar per-day risk of infection (4). The infection rate among travelers to source countries was multiplied by the total person-time at risk for the population of that country using the following formula:

cumulative number of severe MERS-CoV cases in Country $\mathrm{X}=$ severe case rate among travelers to source countries $\times$ country $\mathrm{X}$ person-time $=$

No. severe cases reported among travelers returning from source countries

Annual no. travelers to source countries $x$ average trip length $\times$ epidemic period

$\times$ country X population size $\times 365 \times$ epidemic period

where the epidemic period $=3.33$ years (September 2012-January 2016). We estimated CIs using profilelikelihoods (4). This approach can estimate the cumulative incidence of disease regardless of seasonality in infection rates (5).

We analyzed 11 travel-associated MERS cases, including 6 case-patients from high-income countries (online Technical Appendix, http://wwwnc.cdc.gov/EID/ article/22/10/15-1121-Techapp1.pdf). It has been suggested that MERS-CoV surveillance may be better in high-income countries than in lower-income countries (6). We tested this hypothesis by comparing the frequency of case detection among travelers returning to high-income 
Table 1. Estimated cumulative incidence of severe Middle East respiratory syndrome cases in Middle Eastern source countries calculated on the basis of illnesses among travelers, September 2012-January $2016^{*}$

\begin{tabular}{|c|c|c|c|c|c|}
\hline \multirow[b]{2}{*}{ Traveler origins } & \multicolumn{5}{|c|}{ Estimated no. cases $(95 \% \mathrm{Cl})$} \\
\hline & All countries & Saudi Arabia & Jordan & Qatar & UAE† \\
\hline $\begin{array}{l}\text { Visitors from high-income OECD } \\
\text { countries }\end{array}$ & $3,263(1,297-6,613)$ & $2,269(902-4,599)$ & 483 (192-979) & $163(65-330)$ & $347(138-704)$ \\
\hline All non-Middle Eastern visitors & $1,431(743-2,452)$ & $995(517-1,705)$ & $212(110-363)$ & $72(37-123)$ & $152(79-261)$ \\
\hline
\end{tabular}

countries, as defined by the Organization for Economic Cooperation and Development (http://www/oecd.org/ about/membershipandpartners/), versus lower-income countries (all other non-Middle Eastern countries worldwide). We found that significantly more cases have been identified in high-income settings $(\mathrm{p}<0.001$ by Fisher exact test; online Technical Appendix). Consequently, we produced 2 sets of calculations, 1 using data only from high-income countries and 1 that combined data from all non-Middle Eastern countries. The high-income country analysis does not assume that no cases occurred in lowerincome countries but shows different case detection rates across travelers' home countries.

Using data for 32 high-income countries, we estimated $\approx 3,263$ severe cases (95\% CI 1,297-6,613; Table 1) for all source countries during September 2012-January 2016. We calculated that Saudi Arabia had the largest number of cases $(2,269,95 \%$ CI $902-4,599)$. We estimated $\approx 1,431$ severe cases $(95 \%$ CI $743-2,452)$ when data from highincome and lower-income countries were combined.

We conducted sensitivity analyses in which we included 1) laboratory-confirmed cases among travelers for whom it was unclear in which country they had been infected or if they had been infected by another travel-associated case-patient and 2) probable but non-laboratory-confirmed MERS$\mathrm{CoV}$ cases reported in travelers. These analyses indicated there could have been up to 4,895 severe cases across source countries $(95 \%$ CI $2,352-8,824)$. We also conducted sensitivity analyses to assess the effect of uncertainty of travelers' average length of stay in source countries (Table 2; online Technical Appendix Tables 2, 3). Increases in travelers' assumed lengths of stay produced lower cumulative incidence estimates related to lower estimated infection rates, and decreases in travelers' assumed lengths of stay produced higher case estimates. For example, using data for travelers from high-income nations, a 2-day increase in average length of stay produced estimates of 2,326 severe cases across source countries (95\% CI 924-4,714; online Technical Appendix Table 2), and a 2-day decrease in lengths of stay produced estimates of 5,463 severe cases $(95 \% \mathrm{CI}$ 2,171-11,071).

\section{Conclusions}

We used data on the incidence of MERS among travelers returning from the Middle East to better estimate the occurrence of severe disease in the most affected countries. We estimated that there were $\approx 3,300$ cases of severe disease in the 4 source countries during September 2012 January 2016. This estimate was 2.3 -fold higher than the total number of laboratory-confirmed cases across source countries from September 2012-January 2016.

Using data up to August 2013, Cauchemez et al. estimated the total case count to be 11-fold higher than the number of laboratory-confirmed cases reported across source countries (4). The closer agreement between observed and estimated cases in our analysis is consistent with improvements in surveillance practices across source countries during 2014 (6-8). Our results are also complementary to a serologic study from Saudi Arabia that reported antibodies to MERS-CoV were found in $0.15 \%$ of the population (10). Our study adds information by focusing on severe infections (which are of greatest clinical concern) and providing more up-to-date information by including data from the 2-year period after the serologic samples were collected.

Our estimates were based on a small sample size (11 travel-associated cases) and assumed that travelers and residents

Table 2. Estimated cumulative incidence of severe Middle East respiratory syndrome cases in Middle Eastern source countries calculated on the basis of illnesses among travelers and traveler LOS, September 2012-January 2016*

\begin{tabular}{lccccc}
\hline & \multicolumn{4}{c}{ Estimated no. cases (95\% Cl) } \\
\hline Traveler data & -2 Days LOS & -1 Day LOS & Average LOS $\dagger$ & +1 Day LOS & +2 Days LOS \\
\hline Visitors from high-income OECD & 5,463 & 4,086 & 3,263 & 2,716 & 2,326 \\
countries & $(2,171-11,071)$ & $(1,623-8,280)$ & $(1,297-6,613)$ & $(1,079-5,504)$ & $(924-4,714)$ \\
All non-Middle Eastern visitors & 2,043 & 1,683 & 1,431 & 1,245 & 1,102 \\
& $(1,061-3,499)$ & $(874-2,883)$ & $(743-2,452)$ & $(647-2,132)$ & $(572-1,887)$ \\
\hline
\end{tabular}

*LOS, length of stay; OECD, Organization for Economic Cooperation and Development.

†The average length of stay of travelers from OECD countries in the 4 source countries (Saudi Arabia, Jordan, Qatar, and United Arab Emirates) was estimated to be $5.0 \mathrm{~d}$, and the average length of stay of travelers from all non-Middle Eastern countries in the 4 source countries was estimated to be 6.7 $\mathrm{d}$ (online Technical Appendix, http://wwwnc.cdc.gov/EID/article/22/10/15-1121-Techapp1.pdf). Only the emirates of Abu Dhabi and Dubai were included in United Arab Emirates calculations due to a lack of traveler data on the other 5 emirates; no cases have been reported from these other emirates. 
of the Middle East had similar infection risks. Our sensitivity analyses demonstrated that results are sensitive to travelers' estimated lengths of stay and also showed that estimates of the epidemic size that incorporated data from lower-income countries were $60 \%$ lower than estimates obtained by using data from high-income countries alone. This finding implies different levels of case detection across travelers' home countries or different MERS-CoV exposure between visitors of different nationalities. Additional data (e.g., larger sample size, travel volume, and lengths of stay, stratified by age and immigration status, frequencies of testing, and contact with camels) could provide further estimates.

Public health officials are concerned about MERS$\mathrm{CoV}$, both in the source countries and from exported cases in persons who can seed outbreaks elsewhere $(9,11)$. By better estimating the epidemic size in the Middle East, our results can help guide public health preparedness efforts in source countries and contribute to projections of the number of cases that could occur among travelers $(9,11-13)$.

Dr. O'Hagan is an epidemiologist and transmission modeler at Centers for Disease Control and Prevention. His interests include the combined use of mathematical modeling and epidemiologic studies to better understand the effect of infectious disease risk factors and interventions.

\section{References}

1. WHO MERSs-CoV Research Group. State of knowledge and data gaps of Middle East respiratory syndrome coronavirus (MERS-CoV) in humans. PLoS Curr. 2013;5. http://dx.doi.org/10.1371/currents.out breaks.0bf719e352e7478f8ad85fa30127ddb8

2. World Health Organization. Middle East respiratory syndrome coronavirus (MERS-CoV) - Thailand, January 292016 [cited 2016 Feb 2]. http://www.who.int/csr/don/29-january-2016-mers-thailand/en/

3. Centers for Disease Control and Prevention. Middle East respiratory syndrome (MERS): countries with lab-confirmed MERS cases [cited 2015 Jun 6]. http://www.cdc.gov/coronavirus/ mers/index.html

4. Cauchemez S, Fraser C, Van Kerkhove MD, Donnelly CA, Riley S, Rambaut A, et al. Middle East respiratory syndrome coronavirus: quantification of the extent of the epidemic, surveillance biases, and transmissibility. Lancet Infect Dis. 2014;14:50-6. http://dx.doi.org/10.1016/S1473-3099(13)70304-9

5. Fraser C, Donnelly CA, Cauchemez S, Hanage WP, Van Kerkhove MD, Hollingsworth TD, et al. Pandemic potential of a strain of influenza A (H1N1): early findings. Science. 2009;324:1557-61. http://dx.doi.org/10.1126/science.1176062

6. Saudi Arabia Ministry of Health. Ministry of health updates historical MERS-CoV infection data, 18 Sep 2014 [cited 2016 Jan 26]. http://www.moh.gov.sa/en/CCC/PressReleases/ Pages/Statistics-2014-09-18-002.aspx

7. Saudi Arabia Ministry of Health. Update in statistics: Ministry of Health institutes new standards for reporting of MERS-CoV, June 3, 2014 [cited 2016 Jan 26]. http://www.moh.gov.sa/en/CCC/ PressReleases/Pages/mediastatement-2014-06-03-001.aspx

8. Saudi Arabia Ministry of Health. Case definition and surveillance guidance for MERS-CoV testing in Saudi Arabia-13 May 2014; 1-2 [cited 2016 Feb 1]. http://www.moh.gov.sa/en/CoronaNew/ Regulations/MoHCaseDefinitionMERSCoVVersionMay132014.pdf

9. Khan K, Sears J, Hu VW, Brownstein JS, Hay S, Kossowsky D, et al. Potential for the international spread of Middle East respiratory syndrome in association with mass gatherings in Saudi Arabia. PLoS Curr. 2013:5. http://dx.doi.org/10.1371/currents. outbreaks.a7b70897ac2fa4f79b59f90d24c860b8

10. Müller MA, Meyer B, Corman VM, Al-Masri M, Turkestani A, Ritz D, et al. Presence of Middle East respiratory syndrome coronavirus antibodies in Saudi Arabia: a nationwide, crosssectional, serological study. Lancet Infect Dis. 2015;15:559-64. http://dx.doi.org/10.1016/S1473-3099(15)70090-3

11. Lessler J, Rodriguez-Barraquer I, Cummings DA, Garske T, Van Kerkhove M, Mills H, et al. Estimating potential incidence of MERS-CoV associated with Hajj pilgrims to Saudi Arabia, 2014. PLoS Curr. 2014:6. http://dx.doi.org/10.1371/currents.outbreaks. c5c9c9abd636164a9b6fd4dbda974369

12. Carias C, O’Hagan JJ, Jewett A, Gambhir M, Cohen NJ, Haber Y, et al. Exportations of symptomatic cases of MERS-CoV infection to countries outside the Middle East. Emerg Infect Dis. 2016;22:723-5. http://dx.doi.org/10.3201/eid2204.150976

13. Chowell G, Blumberg S, Simonsen L, Miller MA, Viboud C. Synthesizing data and models for the spread of MERS-CoV, 2013: key role of index cases and hospital transmission. Epidemics. 2014;9:40-51. http://dx.doi.org/10.1016/j.epidem.2014.09.011

Address for correspondence: Justin J. O'Hagan, Centers for Disease Control and Prevention, 1600 Clifton Rd NE, Mailstop A16, Atlanta, GA 30329-4027, USA; email: johagan@cdc.gov

\section{EID SPOTLIGHT TOPIC}

MERS is an illness caused by a virus called Middle East Respiratory Syndrome Coronavirus (MERS-CoV). MERS affects the respiratory system. Most MERS patients developed severe acute respiratory illness with symptoms of fever, cough, and shortness of breath. Health officials first reported the disease in Saudi Arabia in September 2012. Through retrospective investigations, health officials later identified that the first known cases of MERS occurred in Jordan in April 2012. MERS-CoV has spread from people with the virus to others through close contact, such as caring for or living with an infected person.

\section{EMERGING}

INFECTIOUS DISEASES http://wwwnc.cdc.gov/eid/page/mers-spotlight 